\title{
Dark exciton preparation in a quantum dot by a longitudinal light field tuned to higher exciton states
}

\author{
M. Holtkemper, ${ }^{1}$ G. F. Quinteiro $\odot,,^{2, *}$ D. E. Reiter $\odot,{ }^{1, \dagger}$ and T. Kuhn $\oplus^{1, *}$ \\ ${ }^{1}$ Institut für Festkörpertheorie, Universität Münster, Wilhelm-Klemm-Strasse 10, 48149 Münster, Germany \\ ${ }^{2}$ IMIT and Departamento de Física FaCENA, Universidad Nacional del Nordeste, Corrientes, Argentina
}

(Received 6 March 2020; revised 30 November 2020; accepted 11 December 2020; published 11 January 2021)

\begin{abstract}
Several important proposals to use semiconductor quantum dots in quantum information technology rely on the control of the dark exciton ground states, such as dark exciton based qubits with a microsecond lifetime. In this paper, we present an efficient way to occupy the dark exciton ground state by a single short laser pulse. The scheme is based on an optical excitation with a longitudinal field component featured by, e.g., radially polarized beams or certain Laguerre-Gauss or Bessel beams. Utilizing this component, we show within a configuration interaction approach that high-energy exciton states composed of light-hole excitons and higher dark heavy-hole excitons can be addressed. When the higher exciton relaxes, a dark exciton in its ground state is created.
\end{abstract}

DOI: 10.1103/PhysRevResearch.3.013024

\section{INTRODUCTION}

With their discrete energy states, semiconductor quantum dots (QDs) are destined to play an important role in solidstate quantum information technology [1], for example, as sources for single or entangled photons [2-6] or as realizations of qubits [7-9]. For qubits, it is especially promising to use the dark excitons as information storage since they exhibit extraordinary long life and coherence times [10-14]. Furthermore, dark excitons are important intermediate states in some state preparation protocols, e.g., for biexciton [15] or impurity spin preparation [16], and they have been used for the generation of a cluster state of entangled photons [17].

The long lifetime of dark excitons is mainly due to the vanishing or very weak dipole coupling to the light field which, in turn, makes their optical generation challenging. Several workarounds have been developed to overcome this difficulty. By using a nonresonant cw excitation and a subsequent random optical charging of the QD one can form excited biexcitons with various spin configurations. After emission of a photon and subsequent phonon-induced hole relaxation certain spin configurations lead to the formation of a dark exciton [12]. Alternatively, weak mixing between bright and dark excitons can be induced either by applying an external magnetic field $[18,19]$ or by breaking the $C_{2 v}$ symmetry of the QD [20]. This makes a direct optical excitation of the dark exciton possible [21], however, with an oscillator strength which is some orders of magnitude smaller than the absorption into the bright exciton. A desired increase of the optical excitation

\footnotetext{
*gfquinteiro@exa.unne.edu.ar

†Doris.Reiter@uni-muenster.de

Tilmann.Kuhn@uni-muenster.de
}

Published by the American Physical Society under the terms of the Creative Commons Attribution 4.0 International license. Further distribution of this work must maintain attribution to the author(s) and the published article's title, journal citation, and DOI. by a stronger coupling between dark and bright excitons is unevitably associated with a further undesired distortion of the spin character of the dark exciton resulting, e.g., in a decrease in the lifetime.

In this paper, we propose a scheme to circumvent this connection of absorption strength and spin distortion by moving the necessary spin coupling to higher excited states. There the spin coupling can be strong essentially without affecting the pure spin character of the ground states. In detail, our scheme utilizes suitable complex light fields with a strong longitudinal field component. Such field components can excite otherwise optically forbidden light-hole (LH) excitons. Valence-band mixing couples those LH excitons preferentially to higher heavy-hole $(\mathrm{HH})$ excitons involving a dark spin configuration, building strongly mixed higher exciton states. Due to their dark $\mathrm{HH}$ exciton contribution, these states will then relax by phonon emission preferentially into the dark $\mathrm{HH}$ exciton ground state.

Our proposal is related to the findings in Ref. [22] where a small coupling between the dark $\mathrm{HH}$ ground state and a LH exciton with total angular momentum zero was found to enable an optical recombination of the dark exciton ground state by emitting a photon in the in-plane direction with a polarization in the $z$ direction. This recombination was identified as the main relaxation process of the dark ground state. However, the coupling is small, because the lowest LH exciton and the dark $\mathrm{HH}$ ground state are strongly separated in energy. Considering higher excited $\mathrm{HH}$-shells, such as the $d$ shell, we will show that these couplings become strong and enable the proposed efficient scheme to excite the dark HH ground state. Our proposal, thus, complements studies on the great benefits of the use of higher QD exciton states [23-27] or complex light fields beyond Gaussian beams [28,29] for QD based quantum information technology.

The paper is structured as follows: In Sec. II we describe light modes which carry strong components of the required polarization in the QD growth direction. Section III then introduces our QD model and basic selection rules for the transverse and longitudinal light modes. The absorption spectra corresponding to these modes are discussed in Sec. IV. 
Based on this knowledge, we present the excitation scheme for the dark exciton ground states in Sec. V and analyze their dependence on the QD geometry in Sec. VI. Conclusions are given in Sec. VII.

\section{A LIGHT FIELD POLARIZED IN THE GROWTH DIRECTION}

A key element for our excitation process is a component of the light field polarized in the growth direction of the QD sample. Such a polarization could be achieved by a laser entering the QD sample from a cleaved edge $[13,30]$. However, cleaving the sample is often associated with other drawbacks, e.g., by introducing additional surfaces close to the QD. Therefore, it is desirable to use a beam at normal incidence along the growth direction, which has a component of the electric field polarized along the propagation direction. In the following we will call this a longitudinal component. Although such a longitudinal field component is absent in plane wavelike fields and is very small in beams which are well described by the paraxial approximation, it can become dominant close to the beam axis in certain strongly nonparaxial or tightly focused beams, such as certain Laguerre-Gaussian, Bessel or radially polarized beams [31-34]. This dominance can be strong enough to safely neglect all but the longitudinal field components in the region close to the beam center. Such longitudinal components have been successfully used to probe single molecules [32] or to trap metallic Rayleigh particles [33].

To provide a clear and well-defined theoretical description, here we consider Bessel beams, which constitute an exact and complete set of solutions to Maxwell's equations. They are propagation-invariant (nondiffracting) beams. Although strictly speaking Bessel beams have an infinite lateral extension because of their weak decay in the radial direction, approximations of such beams have been realized in various experiments [35-38]. Furthermore, it can be shown that the tight focusing of cylindrically symmetric vector beams typically also results in Bessel functionlike field distributions in the transverse and longitudinal components [31].

Assuming a beam propagating along the $z$ direction with $\mathbf{E}(\mathbf{r}, t)=\frac{1}{2} \tilde{\mathbf{E}}(\mathbf{r}) e^{i\left(q_{z} z-\omega t\right)}+$ c.c., where c.c. denotes the complex conjugate, the electric field of a Bessel beam reads

$$
\begin{aligned}
& \tilde{E}_{x}(\mathbf{r})=i \sigma \tilde{E}_{y}(\mathbf{r})=i \frac{E_{0}}{\sqrt{2}} J_{\ell}\left(q_{r} r\right) e^{i \ell \varphi}, \\
& \tilde{E}_{z}(\mathbf{r})=\sigma \frac{E_{0}}{\sqrt{2}} \frac{q_{r}}{q_{z}} J_{\ell+\sigma}\left(q_{r} r\right) e^{i(\ell+\sigma) \varphi}
\end{aligned}
$$

with the Bessel function of first kind and $\ell$ th order $J_{\ell}$, the electric-field amplitude $E_{0}$, the frequency $\omega$, and the wavevector components in the propagation and the radial directions $q_{z}$ and $q_{r}$, respectively. The latter quantities are related by $q_{z}^{2}+q_{r}^{2}=(n \omega / c)^{2}, n$ being the index of refraction of the medium. The integers $\sigma= \pm 1$ and $\ell=0, \pm 1, \ldots$ characterize the handedness of circular polarization and the orbital angular momentum of the transverse components of the beam, respectively. We consider a CdSe QD with $n=2.8$ [39] and realistic highly nonparaxial (tightly focused) beams with $q_{r} / q_{z}=1[40,41]$.
Since for all Bessel functions $J_{n}$ with $n>0$ the intensity of the beam profile has a minimum around the beam axis (thus, at the position of the QD), there are only two types of beams with a high intensity at the QD: First, beams with $\ell=0$ and $\sigma= \pm 1$, which are characterized by a predominantly transverse field component. Selection rules and spectra of these beams are similar to those of typical Gaussian (or plane wavelike) beams. Second, beams with $\ell= \pm 1$ and $\sigma=$ $\mp 1$, which are characterized by a predominantly longitudinal field component. We note, that one can also superpose the two Bessel beam modes $\ell= \pm 1$ and $\sigma=\mp 1$ to a radially or azimuthally polarized beam where the radially polarized beam also features the pronounced longitudinal field component [31-33] whereas the azimuthally polarized beam remains purely transverse with vanishing intensity at the beam center [31]. In the following we will consider the absorption by both the transverse and the longitudinal field components.

\section{ELECTRONIC STATES AND OPTICAL SELECTION RULES}

We are interested in the optical excitation of a flat selfassembled QD with a diameter of a few nanometers. The height is usually by a factor of 2-4 smaller than the diameter. The confinement of electron and hole states is modeled by a three-dimensional harmonic oscillator potential with confinement lengths $L_{x}, L_{y}$ and $L_{z}$. The electronic states are described within an envelope function formalism. Therein, the wave function is separated into a Bloch part and an envelope part. For the Bloch states, we take into account the $\mathrm{HH}$ valence band with a total angular momentum projection (or pseudospin) of $J_{h}= \pm 3 / 2$, the LH valence band with $J_{h}= \pm 1 / 2$ and the conduction band for electrons with $J_{e}= \pm 1 / 2$. According to the confinement, the envelope states are given by harmonic-oscillator basis functions $\Phi_{a_{x}}(x) \Phi_{a_{y}}(y) \Phi_{a_{z}}(z)$ with quantum numbers $a_{\alpha}=0,1,2, \ldots$. Because of the flat QD structure, the here considered lowest-lying states just increase in the in-plane quantum numbers $a_{x}$ and $a_{y}$. We label the corresponding states via the envelope quantum number sum $a_{x}+$ $a_{y}=0,1,2, \ldots$ by $s, p, d, \ldots(S, P, D, \ldots)$, which also denotes the number of nodal planes perpendicular to the $x y$ plane. For typical QDs, which do not exhibit a too strong in-plane anisotropy, equally labeled states are clustered in energy shells (such as the different states fulfilling $a_{x}+a_{y}=2$, denoted in the following as $d$ shells). If $a_{z} \neq 0$, we add the index $z$ (such as in $p_{z}$ ). We use small letters for electrons and HHs, and capital letters for LHs. Excitons as well as their exciting transitions are labeled by the valence-band to conductionband state involved (e.g., $d \rightarrow s$ denotes the excitation of a $\mathrm{HH}$ from the $d$ shell to an electron in the $s$ shell). The Bloch part of the wave function determines the spin selection rules. For transverse electric fields only electron-hole pairs with a total angular momentum projection of \pm 1 can be excited, i.e., $\mathrm{HH} \pm 1$ excitons with $J_{h}= \pm 3 / 2$ and $J_{e}=\mp 1 / 2$ and $\mathrm{LH} \pm 1$ excitons with $J_{h}= \pm 1 / 2$ and $J_{e}= \pm 1 / 2$. Transitions into the dark $\mathrm{HH} \pm 2$ excitons with $J_{h}= \pm 3 / 2$ and $J_{e}= \pm 1 / 2$ and $\mathrm{LH} \pm 0$ excitons with $J_{h}= \pm 1 / 2$ and $J_{e}=\mp 1 / 2$ are spin forbidden [12]. For longitudinal field components, new spin selection rules apply and the light-hole excitons $\mathrm{LH} \pm 0$ [29] can be excited, whereas $\mathrm{HH} \pm 1, \mathrm{HH} \pm 2$, and $\mathrm{LH} \pm 1$ are 


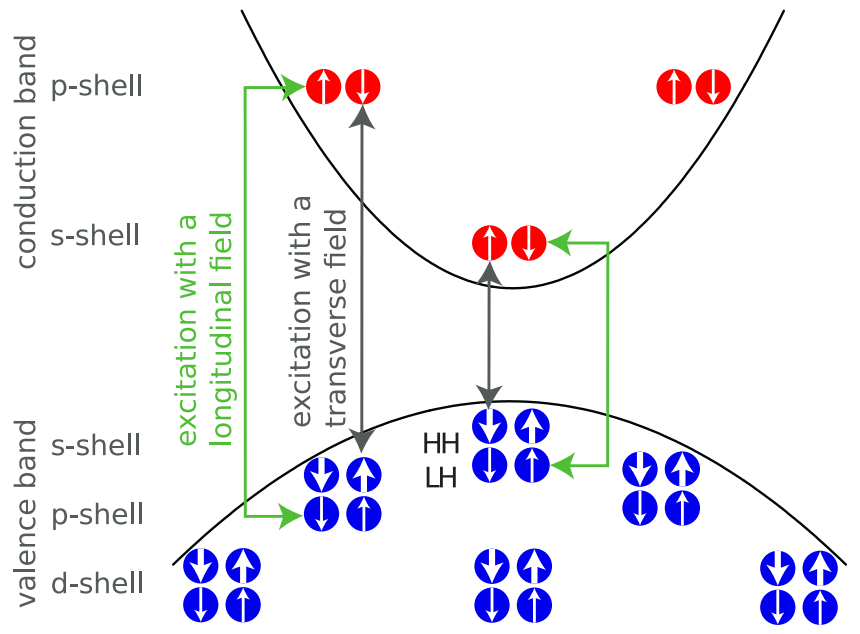

FIG. 1. Sketch of the optical selection rules in a QD. The red dots mark the conduction-band electrons and the blue dots represent the valence-band holes, whereas the spin is indicated by a white arrow (bold for HHs and thin for electrons and LHs). The states are grouped in the usual shells $s, p, d, \ldots$. The dark gray (light green) arrows mark exemplary electron-hole pairs which can be excited by a transverse (longitudinal) field component.

spin forbidden. The transitions between different envelope functions are governed by their overlap integral. Since the confinement lengths for conduction- and valence-band states are taken to be the same, different envelope functions are orthogonal and only transitions between states with the same envelope functions are possible. Figure 1 provides a sketch of exemplary transitions addressable by the transverse (gray arrows) and longitudinal field (light green arrows). The electron and hole states are depicted as red and blue dots, respectively. The white arrows (bold for HHs and thin for electrons and LHs) indicate the different spin states, whereas the groups of two/four states represent the different envelope states.

\section{ABSORPTION SPECTRA FOR THE TRANSVERSE AND LONGITUDINAL MODES}

Using a simple model of uncoupled electron-hole pairs inside the QD, we can calculate the corresponding absorption spectrum, which is shown in Fig. 2(a) for the two types of exciting beams. Here we have considered a QD with a size of $\left(L_{x} \times L_{y} \times L_{z}\right)=(5.4 \times 5.4 \times 2.0) \mathrm{nm}^{3}$ and material parameters for CdSe as in Ref. [26]. The small lines at the bottom mark the positions of all states, which are, in general, multiply degenerate. We find that the corresponding spectra indeed match our expectations. More specifically, for the transverse field (bottom line) we predict the $s \rightarrow s$ and $p \rightarrow p \mathrm{HH} \pm 1$ transitions and the $S \rightarrow s \mathrm{LH} \pm 1$ transition. For the longitudinal field (top line) only the $S \rightarrow s \mathrm{LH} \pm 0$ transition is excited in the given energetic range, however, the oscillator strength is similar to values expected for typical transverse field excitations.

Valence-band mixing and correlation effects induced by Coulomb interaction become important especially for higher exciton states [26]. To get a more realistic description of a QD, we extend the envelope function approximation by includ-
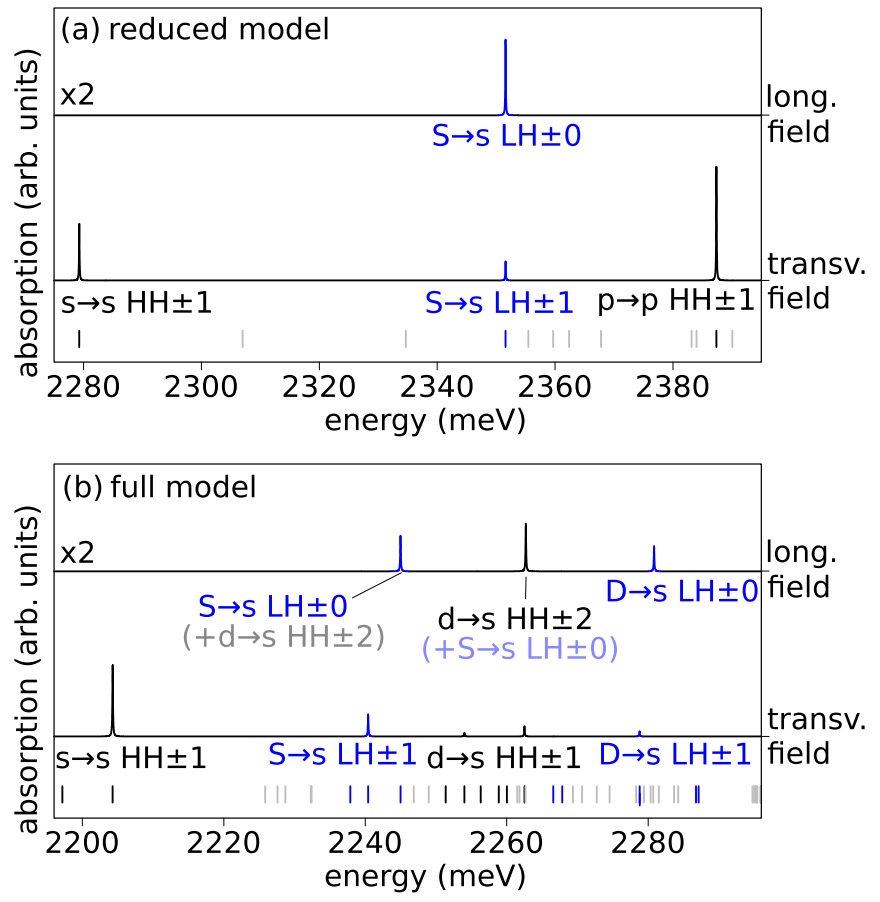

FIG. 2. Optical spectra of a QD in (a) the reduced QD model and (b) the full QD model including Coulomb interaction and valenceband mixing. In each part the bottom line shows the spectrum for the transverse field and the upper line for the longitudinal field. The small lines at the bottom mark the positions of all available states, which in (a) are, in general, multiply degenerate, whereas in (b) many degeneracies are lifted.

ing the direct Coulomb interaction, the short-range exchange Coulomb interaction, as well as valence-band mixing via the Luttinger Hamiltonian within a configuration interaction approach to obtain the exciton eigenstates of the QD. The interactions lead to a strong mixing of the electron-hole pair states. Details of the model, including its limitations, can be found in Ref. [26].

The corresponding spectra are shown in Fig. 2(b) for the two types of excitation discussed above. Below the spectra again all existing states are indicated. Many degeneracies seen in the uncoupled model, associated typically with different spin configurations, are now lifted. For example, at the ground-state transition $(s \rightarrow s)$ a splitting into two levels appears, the lower one reflecting the two dark $\mathrm{HH} \pm 2$, and the upper one reflecting the two bright $\mathrm{HH} \pm 1$ excitons. Both doublets can be further split by breaking the cylindrical symmetry of the QD confinement. The lowest transition involving light holes, $S \rightarrow s$ splits into three levels, a doubly degenerate $\mathrm{LH} \pm 1$ transition as well as two energetically separated states containing the $\mathrm{LH} \pm 0$ excitons, from which one is accessible by the longitudinal field.

Further considerations of the spectra reveal that all the transitions from the reduced model still prevail in the full model, however, at different energies due to the Coulomb shifts. Also, the relative intensities of the lines change. Note that for the sake of clarity we use the same labels for the transitions, now referring to the strongest electron-hole pair state contribution. However, due to the mixing there are, in 


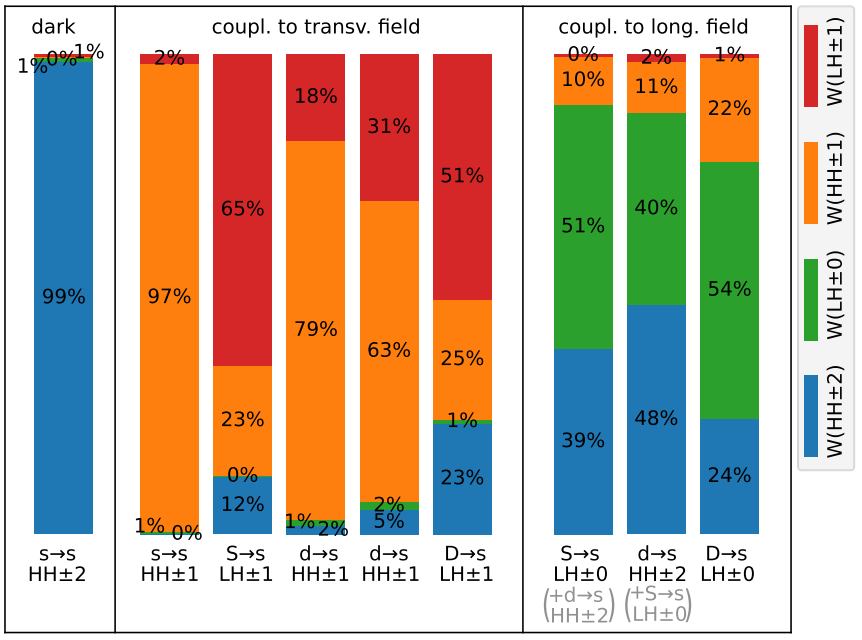

FIG. 3. Different spin contributions of the dark exciton $s \rightarrow$ $s \mathrm{HH} \pm 2$ (not directly accessible by optical excitation) and the excitons excited by the transverse and longitudinal fields for a QD with size $(5.4 \times 5.4 \times 2.0) \mathrm{nm}^{3}$.

general, contributions from other electron-hole pair states. In addition, due to the state mixing several new absorption lines show up. In the considered energetic range these are $d \rightarrow s$ and $D \rightarrow s$ transitions.

It is instructive to understand the spin contributions of the different excitons. We define these spin contributions $W(\mathrm{HH} \pm 2), W(\mathrm{HH} \pm 1), W(\mathrm{LH} \pm 0)$, and $W(\mathrm{LH} \pm 1)$ via the trace over the spatial degrees of freedom, i.e., by summing over all envelope contributions with the respective spin configurations. The spin contributions are displayed in Fig. 3 for the dark ground state exciton and the excitons excited by the transverse and longitudinal fields [see Fig. 2(b)]. We note that although the dark and the bright $\mathrm{HH} s \rightarrow s$ excitons exhibit almost no mixing, all other excitons have pronounced contributions from, at least, two different spin configurations. Especially the predominantly LH eigenstates have strong admixtures of energetically nearby $\mathrm{HH}$ states. This holds, in particular, for those excitons excitable by a longitudinal field. For our QD geometry, the $d \rightarrow s$ HH excitons are energetically close to the $S \rightarrow s$ LH excitons, which leads to a strong mixing of $d \rightarrow s \mathrm{HH} \pm 2$ and $S \rightarrow s \mathrm{LH} \pm 0$. This mixing is so strong, that we include the respective admixture in parentheses within the labeling of the respective eigenstate and call them $S \rightarrow s \mathrm{LH} \pm 0(+d \rightarrow s \mathrm{HH} \pm 2)$ and $d \rightarrow s \mathrm{HH} \pm$ $2(+S \rightarrow s \mathrm{LH} \pm 0)$. In the following, we will focus on these two eigenstates. Please note, that the contributions of the bright spin states do not represent the brightness of the respective exciton since, e.g., envelope selection rules, which are not considered in the discussion of spin contributions, also affect the brightness.

\section{SCHEME TO EXCITE THE DARK EXCITON GROUND STATE}

The strong spin mixing observed for the states $d \rightarrow$ $s \mathrm{HH} \pm 2$ and $S \rightarrow s \mathrm{LH} \pm 0$ in the full QD model builds the basis for our scheme to excite the dark exciton ground state. The scheme is sketched in Fig. 4 and is based on two steps:
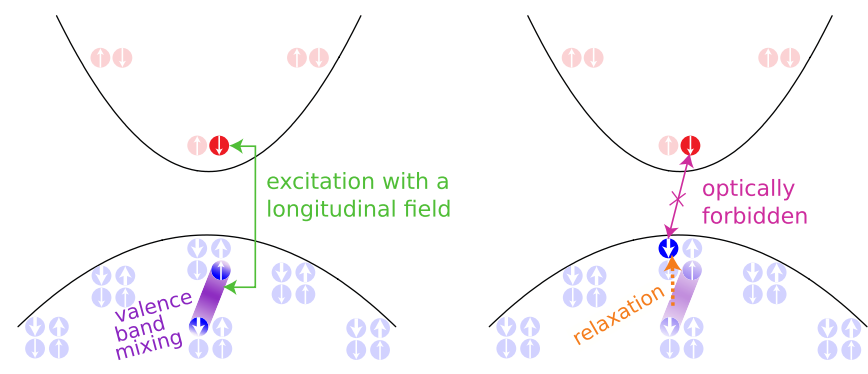

FIG. 4. Proposed scheme for dark exciton generation. QD states in the valence and conduction band are sketched as in Fig. 1. On the left, the purple shaded area indicates the valence-band mixing between a $d$-shell HH and a $s$-shell LH. The green arrow describes the optical excitation of a corresponding exciton by the longitudinal component of a light field. On the right, the relaxation of the mixed hole is illustrated by the orange arrow. The optical recombination of the resulting exciton is forbidden (violet arrow).

(1) As discussed above, the valence-band mixing causes a coupling between $\mathrm{HH} \pm 2$ and $\mathrm{LH} \pm 0$, leading to two mixed eigenstates. The $\mathrm{LH} \pm 0$ spin components of these eigenstates makes them accessible by the longitudinal light field. We will refer to this optical accessibility via the relative brightness $B$, given in units of the oscillator strength of the unmixed $S \rightarrow s \mathrm{LH} \pm 0$ electron-hole pair. In our case [see upper part of Fig. 2(b)], the exciton labeled by $S \rightarrow s \mathrm{LH} \pm 0(+d \rightarrow$ $s \mathrm{HH} \pm 2$ ) has a relative brightness of $B=47 \%$, whereas the exciton labeled by $d \rightarrow s \mathrm{HH} \pm 2(+S \rightarrow s \mathrm{LH} \pm 0)$ has a relative brightness $B=63 \%$. Please note, that these two transitions are well separated in energy from any other transitions so that short laser pulses can be used to selectively address these states.

(2) After excitation, higher excitons tend to relax into lower-lying exciton states, a process mediated by the emission of phonons, which takes place typically on a timescale of a few hundred femtoseconds up to some picoseconds [25], depending on the geometry of the QD. Spin-flip processes via the coupling to nuclear spins [42] occur on a nanosecond timescale and are, therefore, negligible. Because the emission of phonons does not change spins, the spin state of the initial and final eigenstates of a relaxation step need to be similar. To estimate the probability of different relaxation channels, we compare the spin contributions of all possible intermediate states. If there are no equal spin contributions in two states, a relaxation between these states is forbidden.

In Fig. 5 we show the energy level scheme of the lower exciton up to the $d \rightarrow s \mathrm{HH} \pm 2(+S \rightarrow s \mathrm{LH} \pm 0)$ state. The eigenstates are represented by color bars which indicate the respective spin contributions. These color bars are ordered in the vertical direction regarding their energy (measured with respect to the lowest exciton state, i.e., the dark $s \rightarrow s \mathrm{HH} \pm 2$ ground-state exciton) and in the horizontal direction regarding their combined contribution $W(\mathrm{HH} \pm 1)+W(\mathrm{LH} \pm 1)$. We observe that this combined contribution essentially separates the states into two spin groups. This separation is caused by the nature of valence-band mixing. Regarding the spin contributions, the relaxation of a state into other states of the same spin group is preferred (see broad shaded arrows in the background of Fig. 5). However, if the intermediate 


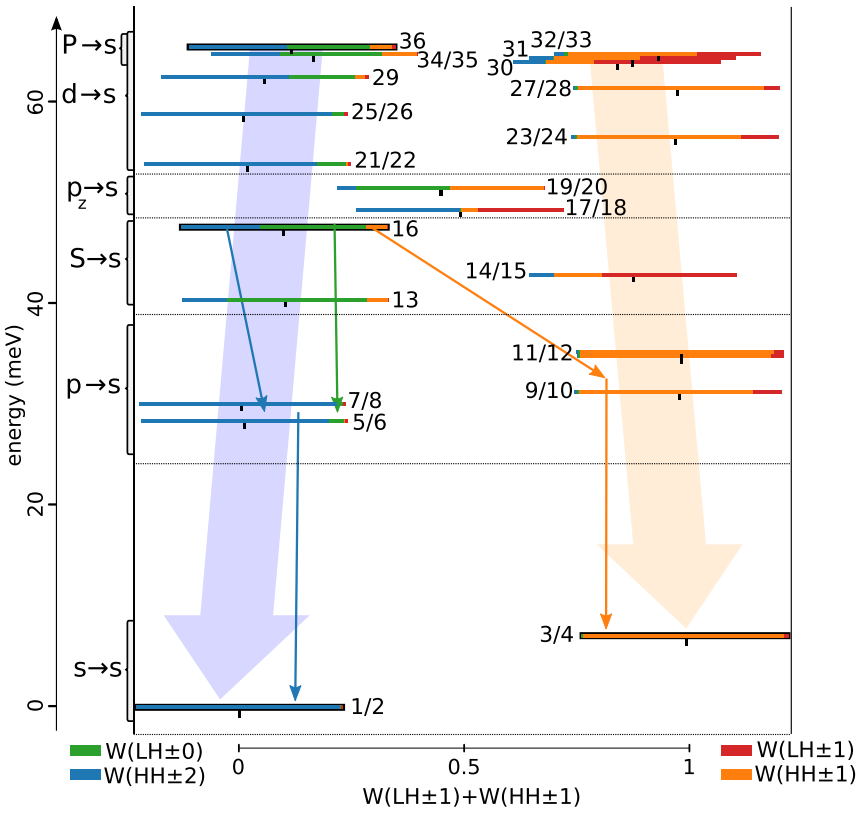

FIG. 5. Color bars representing the contributions $W(\mathrm{HH} \pm$ $2), W(\mathrm{HH} \pm 1), W(\mathrm{LH} \pm 0)$, and $W(\mathrm{LH} \pm 1)$ of the energetically lower exciton states (see small lines below the spectra in Fig. 2). The color bars are ordered by energy in vertical direction and by their combined contribution $W(\mathrm{HH} \pm 1)+W(\mathrm{LH} \pm 1)$ in the horizontal direction. A black frame highlights the color bars representing the two here relevant initial states $S \rightarrow s \mathrm{LH} \pm 0(+d \rightarrow s \mathrm{HH} \pm 2)$ and $d \rightarrow s \mathrm{HH} \pm 2(+S \rightarrow s \mathrm{LH} \pm 0)$, as well as the ground states. The two shaded arrows in the background mark the general relaxation cascades, whereas the thinner arrows mark the possible relaxation paths of the $S \rightarrow s \mathrm{LH} \pm 0(+d \rightarrow s \mathrm{HH} \pm 2)$ state.

states have nonvanishing spin contributions of the other spin group, there is a certain chance for relaxations between the spin groups. Focusing on the relaxation of the $S \rightarrow s \mathrm{LH} \pm$ $0(+d \rightarrow s \mathrm{HH} \pm 2$ ) exciton (state 16 , marked by a black frame), the $\mathrm{HH} \pm 2$ contribution allows a relaxation into the $p \rightarrow s \mathrm{HH} \pm 2$ states (states 5-8), which are of rather pure $\mathrm{HH} \pm 2$ character. These states then relax into the dark ground states (states 1 and 2). In addition, the small $\mathrm{HH} \pm 1$ contribution of state 16 enables a relaxation via the rather pure $p \rightarrow s \mathrm{HH} \pm 1$ spin states (states 9-12) into the bright ground states (states 3 and 4$)$. The ratio of the dark-to-bright spin contribution $R=W(\mathrm{HH} \pm 2) / W(\mathrm{HH} \pm 1)=3.8$ of the initial state 16 can be taken as a rough estimate for the ratio of the final occupation of the dark and bright ground states. The relaxation of the state $d \rightarrow s \mathrm{HH} \pm 2(+S \rightarrow s \mathrm{LH} \pm 0)$ (state 36 ) can be estimated in a similar way leading to $R=4.5$. Here the somehow special states 17-20 might lead to a larger disturbance of the spin state if they are occupied during the relaxation cascade. These states contain a hole state excited in growth direction ( $p_{z}$ state), which leads to a different mixture by valence-band mixing.

Besides these rather qualitative arguments, the question for a more quantitative description of the relaxation channels emerges. The quantitative calculation of the relaxation through tens of states remains challenging, as reliable approaches are still under development. Nonetheless, the

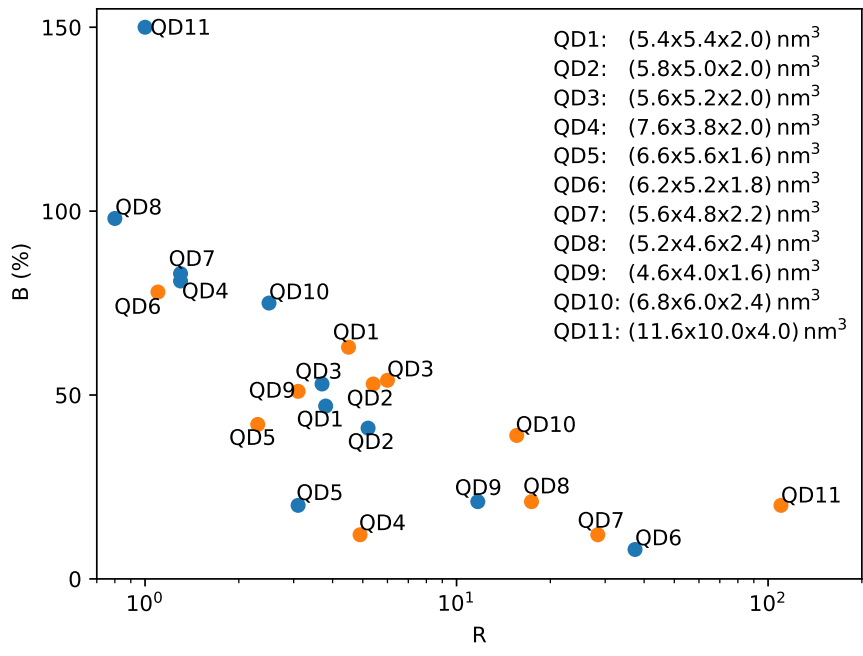

FIG. 6. Relative brightness $B$ and relative dark-to-bright contribution $R$ of the most relevant excitons (see the text) excited by antiparallel Bessel beams in different realistic QD geometries. The energetically lower/higher relevant exciton is given in blue/orange. The numerical values are also given in Table II.

available approaches might give an additional hint on the final occupation of the ground states. Thus, we implemented a model based on acoustic phonon emission and polaron decay (see Appendix A). This approach results in a final ratio of the occupations of the dark to bright exciton ground states of $\tilde{R}=32$ for an initial excitation of the $S \rightarrow s \mathrm{LH} \pm 0(+d \rightarrow$ $s \mathrm{HH} \pm 2)$ exciton and a ratio of $\tilde{R}=4$ for an initial excitation of the $d \rightarrow s \mathrm{HH} \pm 2(+S \rightarrow s \mathrm{LH} \pm 0)$ exciton. Here we want to emphasize that these numbers should be treated with some care because, for example, multiphonon processes have been neglected which might, in particular, be relevant where single-phonon processes lead to a bottleneck in the relaxation process. However, it is not expected that such extensions qualitatively change the relaxation paths. Therefore, $\tilde{R}$ provides an additional hint to the preferred final occupation of the dark exciton ground state. When comparing the two estimates $R$ and $\tilde{R}$, we find that there may be quantitative differences; however, both numbers indicate a dominant relaxation into the dark exciton ground states starting from both initial states 16 and 36 exhibiting the strong $S \rightarrow s \mathrm{LH} \pm 0$ and $d \rightarrow s \mathrm{HH} \pm 2$ mixing, which further supports the viability of our proposal.

\section{DEPENDENCE ON THE QD GEOMETRY}

In the above study, we considered a geometry where the mixture between $S \rightarrow s \mathrm{LH} \pm 0$ and $d \rightarrow s \mathrm{HH} \pm 2$ turned out to be maximal. The question arises whether our proposed excitation scheme crucially depends on the geometry parameters and might, therefore, just be relevant for very specific QDs. To rule out this possibility, we have performed a parameter study as in Ref. [26] on a variety of realistic QD geometries. In addition to the cylindrically symmetric QD discussed up to now (labeled as QD1 in the following) we studied ten different geometries QD2-QD11 with confinement lengths $L_{x}, L_{y}$, and $L_{z}$ as given in Fig. 6 representing QDs with varying volume, thickness, and in-plane asymmetry. In agreement with Ref. [26], in the diagonalization procedure 
(a)

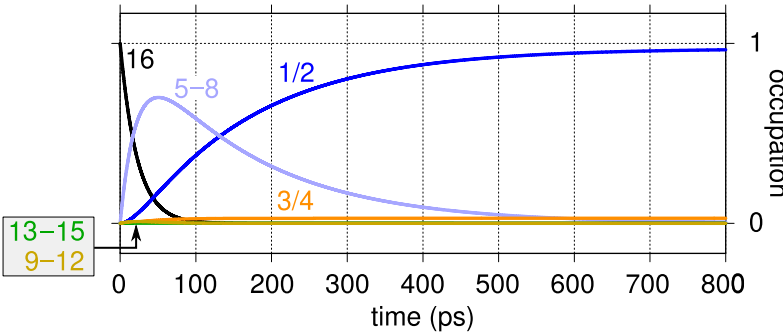

(b)

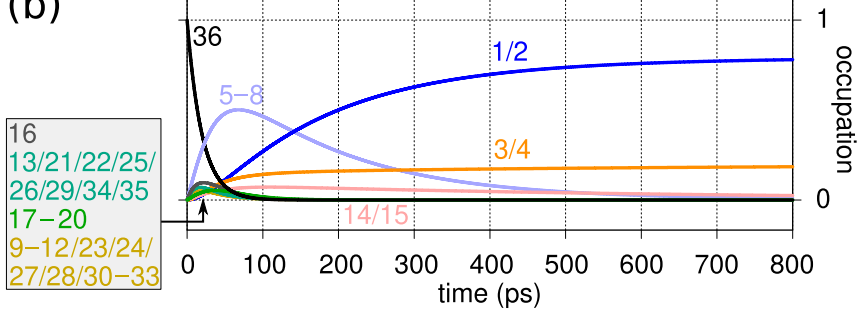

FIG. 7. Time line of the occupation dynamics after an excitation of (a) state 16 and (b) state 36 in QD1. The definition of the states is given in Fig. 5. Each curve refers to the occupation of a single state or the sum of the occupations of a group of state as indicated by the state numbers in the respective color of the curve. The results are obtained based on the explicit model discussed in Appendix A.

basis functions with quantum numbers $\left(a_{x}, a_{y}\right.$, and $\left.a_{z}\right)$ up to $(5,5,3)$ for holes and $(3,3,3)$ for electrons and a maximal quantum-number sum of $a_{x}^{\text {hole }}+a_{x}^{\text {elec. }}+a_{y}^{\text {hole }}+a_{y}^{\text {elec. }}+$ $2\left(a_{z}^{\text {hole }}+a_{z}^{\text {elec. }}\right)=7$ were taken into account. For QD1 and QD2 we even increased the quantum numbers up to $(7,7,7)$ for both electrons and holes and to a maximal quantumnumber sum of $a_{x}^{\text {hole }}+a_{x}^{\text {elec. }}+a_{y}^{\text {hole }}+a_{y}^{\text {elec. }}+a_{z}^{\text {hole }}+a_{z}^{\text {elec. }}=$ 7 , but we observed no significant differences for the states relevant for our scheme, demonstrating the good convergence.

For each geometry, we considered two eigenstates: The one mainly consisting of $S \rightarrow s \mathrm{LH} \pm 0$ spin contributions and the brightest eigenstate mainly consisting of dark $\mathrm{HH} \pm$ 2 spin contributions. In Fig. 6 the obtained values of the relative brightness $B$ are plotted versus the dark-to-bright ratio $R=W(\mathrm{HH} \pm 2) / W(\mathrm{HH} \pm 1)$ between $\mathrm{HH} \pm 2$ and $\mathrm{HH} \pm 1$ spin contribution. The energetically lower (higher) of the two considered exciton states is plotted in blue (orange).

We observe rather large variations in the brightness from $8 \%$ to $150 \%$ and in the dark-to-bright ratio from slightly below 1 up to about 100. Regarding the lowest values, it should be noted that the minimal observed brightness $B=8 \%$ is still several orders of magnitude stronger than a direct excitation of the dark exciton [21]. Taking $R$ as a rough estimate for the final occupation of the ground states, for the lowest values $R \approx 1$ we would get a $50 \%$ chance to occupy the dark exciton. Thus, an excitation of these states would still have a comparable efficiency as an excitation into the wetting layer [43]. However, here we excite a state already within the QD and with a faster and more direct relaxation into the ground state. Despite these worst-case scenarios, some geometries provide states with quite appealing combinations, such as $R=110$ and $B=20 \%$ (QD11) or $R=15.6$ and $B=39 \%(\mathrm{QD} 10)$.

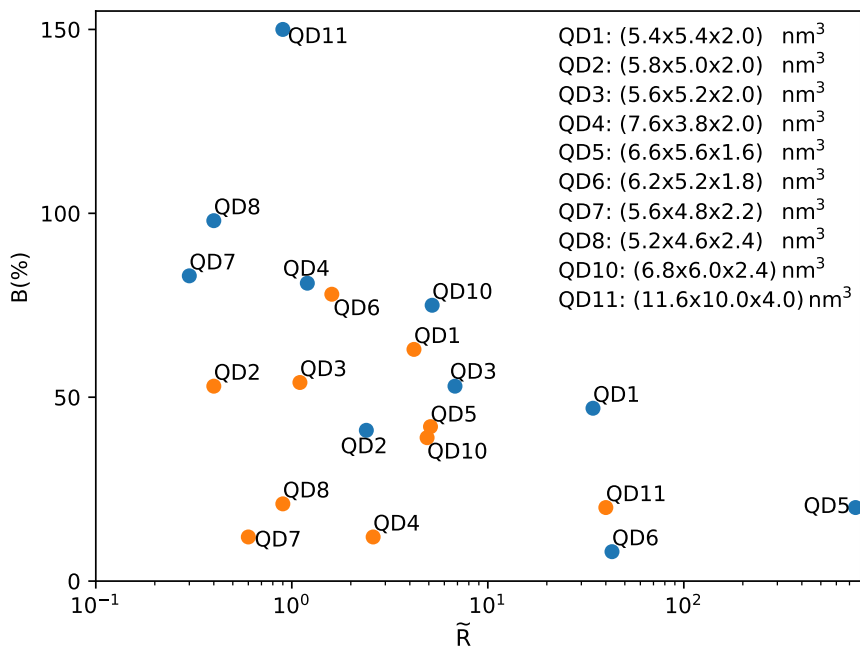

FIG. 8. Relative brightness $B$ and relative final occupation of dark/bright ground state $\tilde{R}$ obtained from the relaxation model for the same excitons as in Fig. 6 in different realistic QD geometries. The energetically lower/higher relevant exciton is given in blue/orange.

Interestingly, we find a group of QDs (QD1, QD2, and QD3) for which both considered exciton states exhibit similar values for $B$ and $R$ in the intermediate ranges of $B \approx 50 \%$ and $R \approx 5$. For another group (QD6, QD7, QD8, and QD11) one of the two states has a rather low $R \approx 1$ but a high value of the brightness $B \gtrsim 80 \%$ whereas the other state has a rather low brightness $B \approx 10-20 \%$ but high dark-to-bright ratios $R \gtrsim$ 20. In a QD from the latter class one might, therefore, choose between a highly efficient optical excitation with a roughly equal occupation of bright and dark excitons or a somewhat less efficient optical excitation for which more than $95 \%$ of the generated occupation is expected to be in the dark state.

An alternative way to estimate the final occupations is provided by the explicit phonon-mediated decay model introduced above and discussed in Appendix A. The dark-to-bright ratio $\tilde{R}$ obtained from this model is taken as the horizontal axis of Fig. 8 in Appendix A whereas the vertical axis again displays the brightness $B$. When comparing the values of $R$ and $\tilde{R}$ for a specific QD there are typically some quantitative differences as already discussed above for the exemplary QD1, however, the overall shapes of Figs. 8 and 6 are similar supporting again the suitability of our proposal.

Our proposal for an efficient preparation of the dark exciton is based on the idea to use the strong coupling between dark and bright spin configurations of energetically higher states to achieve an efficient optical excitation, whereas the dark exciton ground state essentially keeps its pure spin configuration and, thus, its long lifetime. Indeed, we found many examples of QDs in which the brightness of the relevant higher exciton states is between $10 \%$ and $100 \%$ of the unmixed, bright $\mathrm{LH}$ exciton whereas providing a dominant relaxation into the dark exciton ground state. However, it should be kept in mind that the same coupling mechanisms also give rise to a coupling of the dark exciton ground states to the bright $\mathrm{LH} \pm 0$ states, which will increase their coupling to light and reduce their lifetime. Therefore, we have calculated the relative brightness $B_{\mathrm{dgs}}$ of the the dark exciton ground state with respect to the 


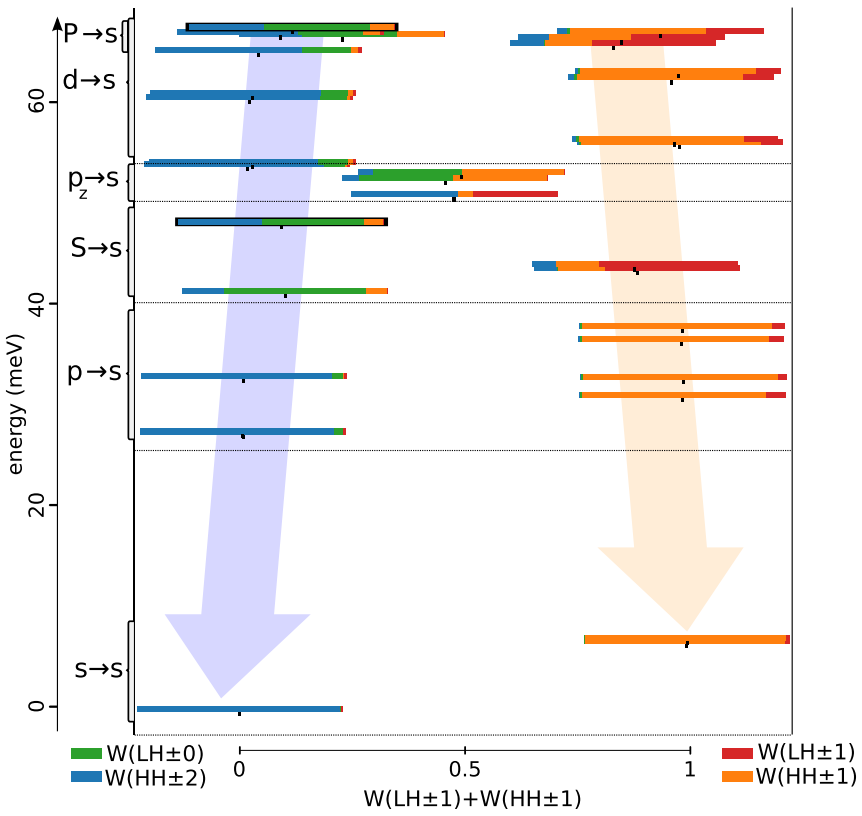

FIG. 9. Same as Fig. 5 but for QD2 with a geometry of $(5.8 \times$ $5.0 \times 2.0) \mathrm{nm}^{3}$.

unmixed bright LH exciton for our QDs. The results are given in the last column of Table II. We obtain typical values around $0.02 \%$, which is, thus, orders of magnitude smaller than the brightness of the here relevant energetically higher dark exciton states. However, we can also identify some trends: The brightness of the dark exciton ground state increases with increasing in-plane elongation (up to $B_{\mathrm{dgs}}=0.5 \%$ for QD4) and height-to-diameter ratio (up to $B=0.05 \%$ for QD8), thus, a flat and close to cylindrically symmetric QD is the best geometry for a pure dark spin configuration of the dark exciton ground state. Please note that due to the inversion symmetry of all our structures the brightness of the dark exciton results only from the coupling to a light field polarized in the $z$ direction, which is the quantity we discuss here. In structures with a broken inversion symmetry in both the growth and the inplane direction, there will be an additional contribution to the brightness resulting from the coupling to in-plane polarized light. The strength of this additional component will strongly depend on the degree of the symmetry breaking.

\section{CONCLUSION}

In conclusion, we have proposed a viable scheme to initialize the dark exciton using a single laser pulse at normal incidence. The scheme has the potential to provide much higher efficiencies than other excitation schemes. Due to long lifetimes, such dark excitons are useful for information storage in quantum technologies. Our scheme utilizes light beams with a pronounced longitudinal component tuned in resonance to certain higher exciton states. These states are characterized by a strong valence-band mixing between optically active excitons with $\mathrm{LH} \pm 0$ spin configurations and higher dark excitons with $\mathrm{HH} \pm 2$ spin configurations, which enables a large coupling to the light field and, at the same time, an ultrafast relaxation path into the dark exciton ground state. We have

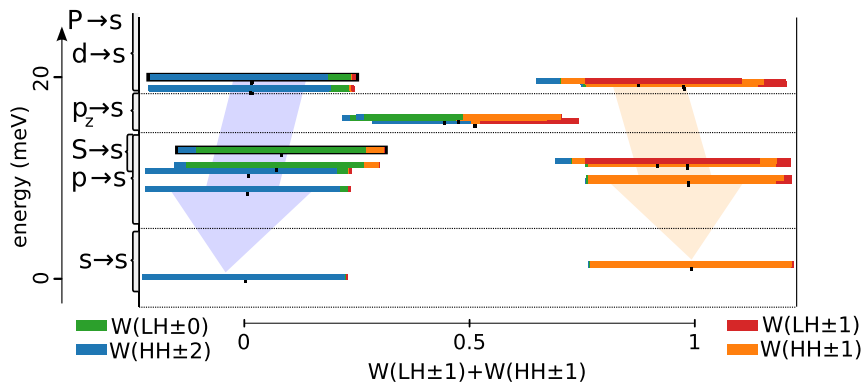

FIG. 10. Same as Fig. 5 but for QD11 with a geometry of $(11.6 \times$ $10.0 \times 4.0) \mathrm{nm}^{3}$.

shown in a parameter study using a configuration interaction approach, that our findings are not specific to a certain QD geometry but appear rather generically in a wide range of QD geometries. In particular, it is known that the strength of valence-band mixing can be adjusted by strain tuning [30], which could provide an additional tool for further optimizing the mixing between bright LH excitons and dark HH excitons. Finally we want to remark that the calculations have been performed for CdSe material parameters. However, the structure of the valence-band mixing, which is the most important contribution here, is determined by the crystal structure, i.e., in the present case by the zinc blende structure. Therefore, we expect qualitatively similar features in QDs made from other zinc blende materials.

\section{ACKNOWLEDGMENT}

G.F.Q. thanks the ONRG for financial support through NICOP Grant No. N62909-18-1-2090.

\section{APPENDIX A: ESTIMATE OF THE RELAXATION CHANNELS}

To model the relaxation rates via acoustic phonons, we implemented a model as described in Ref. [44], Sec. VI. Here we assume zero temperature. To account for the relaxation via longitudinal optical (LO) phonons, we add a relaxation rate deduced from a polaron decay model following Ref. [45]. To adapt the polaron decay times to our otherwise purely electronic model, we consider for each possible relaxation step just the initial and final excitonic eigenstates. Regarding these two excitonic states, we calculate the respective polaron states and use the relaxation time of the polaron closer to the respective initial excitonic state as the relaxation time between the two purely excitonic levels. The rates are then treated in a rate equation model to calculate the final occupation of dark and bright exciton ground states (the relaxation from bright to dark ground state is negligible). Such calculations are numerically quite demanding and typically just performed for single transitions (and not for the here relevant hundreds of possible single relaxations). Consequently, we reduce our basis for the calculation of the state mixtures to just slightly over 200 basis states, however, we keep the energies of the individual states as they are in the main calculation presented, e.g., in Fig. 2(b). We use the material parameters for $\mathrm{CdSe}$ : static dielectric constant $\epsilon_{r}=9.2$ [46], density $\rho=5.81 \mathrm{~g} / \mathrm{cm}^{3}$ [47], lattice 


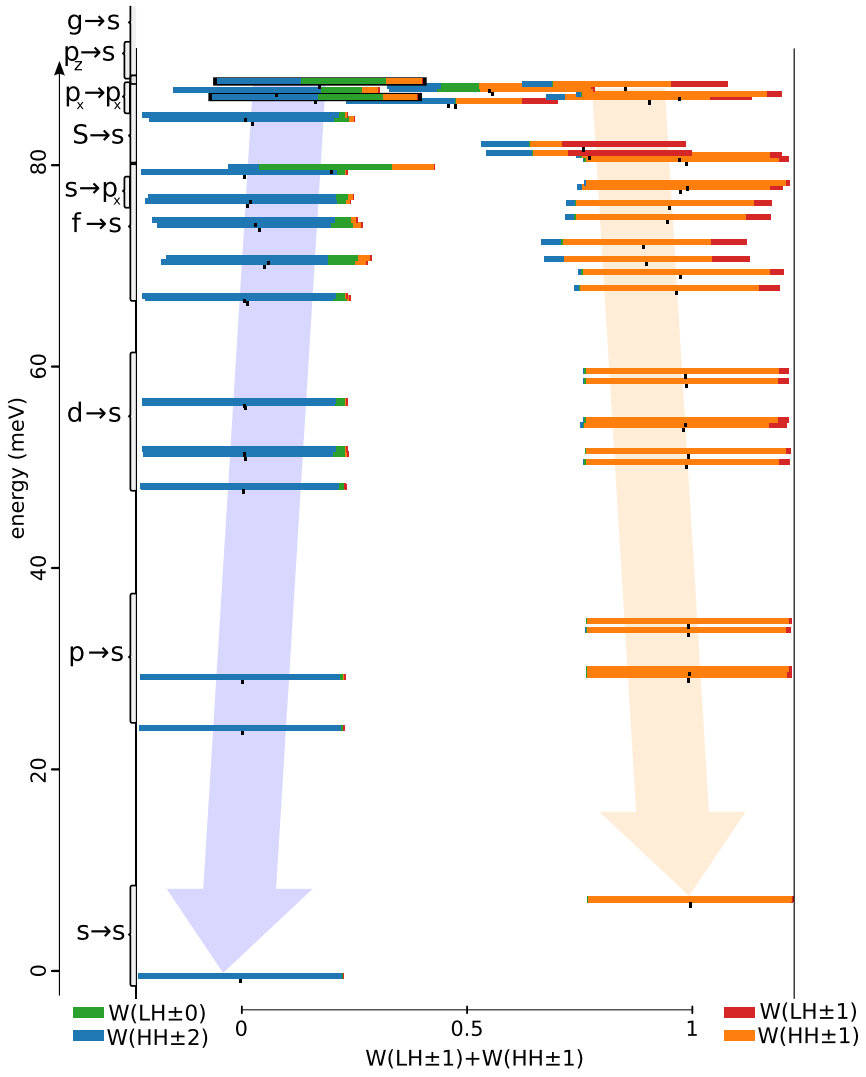

FIG. 11. Same as Fig. 5 but for QD5 with a geometry of $(6.6 \times$ $5.6 \times 1.6) \mathrm{nm}^{3}$.

constant $a_{\text {lat }}=0.6077 \mathrm{~nm}$ [47], sound velocity longitudinal acoustic (LA) phonons $c_{\mathrm{LA}}=3856 \mathrm{~m} / \mathrm{s}$ [47], sound velocity transverse acoustic (TA) phonons $c_{\mathrm{TA}}=1521 \mathrm{~m} / \mathrm{s}$ [47], deformation potential (DP) constant holes $D_{h}^{\mathrm{DP}}=3 \mathrm{eV}$ [48], deformation potential constant electrons $D_{e}^{\mathrm{DP}}=-6 \mathrm{eV}$ [48], piezoelectric constant $e_{14}=0.2 \mathrm{C} / \mathrm{m}^{2}$ [49], band center energy LO phonons $\hbar \omega_{\mathrm{LO}}=25 \mathrm{meV}$ [50], band edge energy LO phonons $\hbar \omega_{\mathrm{LO}}=23 \mathrm{meV}$, high-frequency dielectric constant $\epsilon_{\infty}=5.8$ [51], Grüneisen constant LA phonons $\gamma_{\text {LA }}=1.1$ [52], Grüneisen constant TA phonons $\gamma_{\mathrm{TA}}=3.2$ [53].

Exemplary results of this model are given in Fig. 7, which shows the occupation dynamics after an excitation of the

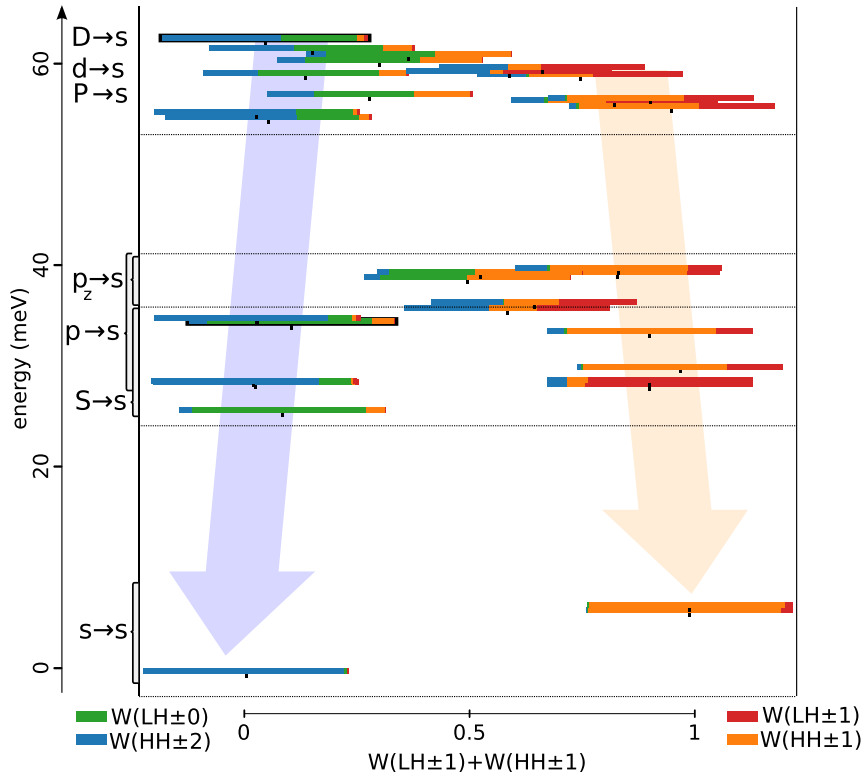

FIG. 12. Same as Fig. 5, but for QD8 with a geometry of $(5.2 \times$ $4.6 \times 2.4) \mathrm{nm}^{3}$.

two relevant states (states 16 and 36) in QD1. The results of this explicit model fit well to the quantitative arguments from the spin considerations around Fig. 5: Starting from state 16 [Fig. 7(a)], the main relaxation goes via states 58 into the dark exciton ground states $1 / 2$. A small part of the occupation, however, goes through states 9-12 into the bright exciton ground states $3 / 4$. Starting from state 36 [Fig. 7(b)], the relaxation cascade is similar, however, due to the larger number of intermediate states, there is a higher chance that also relaxation processes into the bright spin group occur.

We want to emphasize, that these calculations should be taken with some care and are just performed to provide another estimate on a preferred occupation of the dark exciton ground state after excitation of some suitable states. In fact, the polaron model induces a strong resonancelike energetic selection rule for relaxation processes. It can be expected that in models including, e.g., multiphonon pro-

TABLE I. Different spin contributions of the dark exciton and the ones excited by the transverse and longitudinal fields for a QD with size $(5.4 \times 5.4 \times 2.0) \mathrm{nm}^{3} . R$ is the ratio of dark-to-bright spin contributions, and $B$ is the relative brightness of the transitions.

\begin{tabular}{|c|c|c|c|c|c|c|c|}
\hline \multirow{2}{*}{$\begin{array}{l}\text { Coupling } \\
\text { to light }\end{array}$} & \multirow[b]{2}{*}{ Transition } & \multicolumn{4}{|c|}{ Spin contributions } & \multirow[b]{2}{*}{$R$} & \multirow[b]{2}{*}{$B$} \\
\hline & & $\mathrm{HH} \pm 2:$ & $\mathrm{HH} \pm 1:$ & $\mathrm{LH} \pm 0:$ & $\mathrm{LH} \pm 1$ & & \\
\hline Dark & $s \rightarrow s$ & 0.99: & 0.00: & 0.01: & 0.01 & & \\
\hline $\begin{array}{l}\text { Transverse } \\
\text { Field }\end{array}$ & $\begin{array}{l}s \rightarrow s \\
S \rightarrow s \\
d \rightarrow s \\
d \rightarrow s \\
D \rightarrow s\end{array}$ & $\begin{array}{l}0.00: \\
0.12: \\
0.02: \\
0.05: \\
0.23:\end{array}$ & $\begin{array}{l}0.97: \\
0.23: \\
0.79: \\
0.63: \\
0.25:\end{array}$ & $\begin{array}{l}0.01: \\
0.00: \\
0.01: \\
0.02: \\
0.01:\end{array}$ & $\begin{array}{l}0.02 \\
0.65 \\
0.18 \\
0.31 \\
0.51\end{array}$ & $\begin{array}{l}0.0 \\
0.5 \\
0.0 \\
0.1 \\
0.9\end{array}$ & $\begin{array}{c}126 \% \\
39 \% \\
7 \% \\
18 \% \\
9 \%\end{array}$ \\
\hline $\begin{array}{l}\text { Longitudinal } \\
\text { field }\end{array}$ & $\begin{array}{l}S \rightarrow s \\
d \rightarrow s \\
D \rightarrow s\end{array}$ & $\begin{array}{l}0.39: \\
0.48: \\
0.24\end{array}$ & $\begin{array}{l}0.10: \\
0.11: \\
0.22:\end{array}$ & $\begin{array}{l}0.51: \\
0.40: \\
0.54:\end{array}$ & $\begin{array}{c}0.00 \\
0.02 \\
0.01:\end{array}$ & $\begin{array}{l}3.8 \\
4.5 \\
1.1\end{array}$ & $\begin{array}{l}47 \% \\
63 \% \\
33 \%\end{array}$ \\
\hline
\end{tabular}


TABLE II. Most relevant (see text) exciton states excited by antiparallel Bessel beams in different realistic QD geometries. For each state, we provide the different spin contributions, relative brightness $B$, relative spin contribution $R$, and relative final occupation of dark-to-bright ground states $\tilde{R}$ obtained from the relaxation model. The rightmost column gives the brightness $B_{\mathrm{dgs}}$ of the dark exciton ground state for comparison.

\begin{tabular}{|c|c|c|c|c|c|c|c|c|c|}
\hline \multirow{2}{*}{$\begin{array}{l}\text { QD } \\
\text { number }\end{array}$} & \multirow{2}{*}{$\begin{array}{l}\text { Geometry } \\
\text { in }\left(\mathrm{nm}^{3}\right)\end{array}$} & \multicolumn{4}{|c|}{ Spin contributions } & \multirow[b]{2}{*}{$B$} & \multirow[b]{2}{*}{$R$} & \multirow[b]{2}{*}{$\tilde{R}$} & \multirow[b]{2}{*}{$B_{\mathrm{dgs}}$} \\
\hline & & $\mathrm{HH} \pm 2:$ & $\mathrm{HH} \pm 1:$ & $\mathrm{LH} \pm 0$ & $\mathrm{LH} \pm 1$ & & & & \\
\hline QD1 & $5.4 \times 5.4 \times 2.0$ & $\begin{array}{l}0.39: \\
0.48:\end{array}$ & $\begin{array}{l}0.10: \\
0.11:\end{array}$ & $\begin{array}{l}0.51: \\
0.40:\end{array}$ & $\begin{array}{l}0.00 \\
0.02\end{array}$ & $\begin{array}{l}47 \% \\
63 \%\end{array}$ & $\begin{array}{l}3.8 \\
4.5\end{array}$ & $\begin{array}{c}34.4 \\
4.2\end{array}$ & 0 \\
\hline QD2 & $5.8 \times 5.0 \times 2.0$ & $\begin{array}{l}0.45: \\
0.52:\end{array}$ & $\begin{array}{l}0.09: \\
0.10:\end{array}$ & $\begin{array}{l}0.46: \\
0.37:\end{array}$ & $\begin{array}{l}0.01 \\
0.02\end{array}$ & $\begin{array}{l}41 \% \\
53 \%\end{array}$ & $\begin{array}{l}5.2 \\
5.4\end{array}$ & $\begin{array}{l}2.4 \\
0.4\end{array}$ & $0.02 \%$ \\
\hline QD3 & $5.6 \times 5.2 \times 2.0$ & $\begin{array}{l}0.37: \\
0.55:\end{array}$ & $\begin{array}{l}0.10: \\
0.09:\end{array}$ & $\begin{array}{l}0.52: \\
0.35:\end{array}$ & $\begin{array}{l}0.00 \\
0.02\end{array}$ & $\begin{array}{l}53 \% \\
54 \%\end{array}$ & $\begin{array}{l}3.7 \\
6.0\end{array}$ & $\begin{array}{l}6.8 \\
1.1\end{array}$ & $0.005 \%$ \\
\hline QD4 & $7.6 \times 3.8 \times 2.0$ & $\begin{array}{l}0.17: \\
0.60:\end{array}$ & $\begin{array}{l}0.13: \\
0.12:\end{array}$ & $\begin{array}{l}0.69: \\
0.27:\end{array}$ & $\begin{array}{l}0.00 \\
0.01\end{array}$ & $\begin{array}{l}81 \% \\
12 \%\end{array}$ & $\begin{array}{l}1.3 \\
4.9\end{array}$ & $\begin{array}{l}1.2 \\
2.6\end{array}$ & $0.5 \%$ \\
\hline QD5 & $6.6 \times 5.6 \times 1.6$ & $\begin{array}{l}0.52: \\
0.41:\end{array}$ & $\begin{array}{l}0.16: \\
0.17:\end{array}$ & $\begin{array}{l}0.32: \\
0.41:\end{array}$ & $\begin{array}{l}0.00 \\
0.00\end{array}$ & $\begin{array}{l}20 \% \\
42 \%\end{array}$ & $\begin{array}{l}3.1 \\
2.3\end{array}$ & $\begin{array}{c}751.7 \\
5.1\end{array}$ & $0.005 \%$ \\
\hline QD6 & $6.2 \times 5.2 \times 1.8$ & $\begin{array}{l}0.83: \\
0.23:\end{array}$ & $\begin{array}{l}0.02: \\
0.21:\end{array}$ & $\begin{array}{l}0.14: \\
0.55:\end{array}$ & $\begin{array}{l}0.01 \\
0.01\end{array}$ & $\begin{array}{c}8 \% \\
78 \%\end{array}$ & $\begin{array}{c}37.4 \\
1.1\end{array}$ & $\begin{array}{c}43.0 \\
1.6\end{array}$ & $0.01 \%$ \\
\hline QD7 & $5.6 \times 4.8 \times 2.2$ & $\begin{array}{l}0.16: \\
0.77:\end{array}$ & $\begin{array}{l}0.12: \\
0.03:\end{array}$ & $\begin{array}{l}0.72: \\
0.19:\end{array}$ & $\begin{array}{l}0.00 \\
0.02\end{array}$ & $\begin{array}{l}83 \% \\
12 \%\end{array}$ & $\begin{array}{c}1.3 \\
28.4\end{array}$ & $\begin{array}{l}0.3 \\
0.6\end{array}$ & $0.03 \%$ \\
\hline QD8 & $5.2 \times 4.6 \times 2.4$ & $\begin{array}{l}\text { 0.09: } \\
0.58:\end{array}$ & $\begin{array}{l}0.11: \\
0.03:\end{array}$ & $\begin{array}{l}0.80: \\
0.37:\end{array}$ & $\begin{array}{l}0.00 \\
0.02\end{array}$ & $\begin{array}{l}98 \% \\
21 \%\end{array}$ & $\begin{array}{c}0.8 \\
17.4\end{array}$ & $\begin{array}{l}0.4 \\
0.9\end{array}$ & $0.05 \%$ \\
\hline QD9 & $4.6 \times 4.0 \times 1.6$ & $\begin{array}{l}0.64: \\
0.43:\end{array}$ & $\begin{array}{l}0.05: \\
0.14:\end{array}$ & $\begin{array}{l}0.30: \\
0.41:\end{array}$ & $\begin{array}{l}0.01 \\
0.01\end{array}$ & $\begin{array}{l}21 \% \\
51 \%\end{array}$ & $\begin{array}{c}11.7 \\
3.1\end{array}$ & & $0.01 \%$ \\
\hline QD10 & $6.8 \times 6.0 \times 2.4$ & $\begin{array}{l}0.27: \\
0.69:\end{array}$ & $\begin{array}{l}0.11: \\
0.04:\end{array}$ & $\begin{array}{l}0.62: \\
0.25:\end{array}$ & $\begin{array}{l}0.00 \\
0.02\end{array}$ & $\begin{array}{l}75 \% \\
39 \%\end{array}$ & $\begin{array}{c}2.5 \\
15.6\end{array}$ & $\begin{array}{l}5.2 \\
4.9\end{array}$ & $0.02 \%$ \\
\hline QD11 & $11.6 \times 10.0 \times 4.0$ & $\begin{array}{l}0.08: \\
0.87:\end{array}$ & $\begin{array}{l}0.09: \\
0.01:\end{array}$ & $\begin{array}{l}0.83: \\
0.11:\end{array}$ & $\begin{array}{l}0.00 \\
0.02\end{array}$ & $\begin{array}{c}150 \% \\
20 \%\end{array}$ & $\begin{array}{c}1.0 \\
110.0\end{array}$ & $\begin{array}{c}0.9 \\
40.0\end{array}$ & $0.04 \%$ \\
\hline
\end{tabular}

cesses or other scattering mechanisms these sharp resonances will be broadened. However, the development of such more refined relaxation models is beyond the scope of the present paper.

In the actual model, the sharp resonances give rise to two artifacts: First, the calculations typically underestimate the total relaxation rates as it is clearly visible in the timescale of Fig. 7 (which is about two orders of magnitude larger than typically seen in experiments [25]). This problem becomes especially clear for the smallest of the considered QD geometries (QD9) where the energetic level spacing is so large that we get no relaxation at all. This is the reason why QD9 is not included in Fig. 8. Second, small variations in the properties of the QD might result in rather large changes of the relaxation channel if energetic resonances are met. Fortunately, the energy dependence shows no general trend favoring a higher final contribution of the bright or dark ground state. In fact, we assume that its contribution averages out when multiple different QDs are considered. Consequently, we consider the results for several QD geometries in Fig. 8, which shows in analogy to Fig. 6 the brightness $B$ compared to the relative final occupation of dark to bright ground state $\tilde{R}$. The results support the general trend already found in the estimate via the factor $R$ (see Fig. 6). Just random differences for some single states occur. Considering the fact that we compare the results of completely different models, the general trend is surprisingly similar.

\section{APPENDIX B: SPIN CONTRIBUTIONS FOR OTHER QD GEOMETRIES}

Figure 5 represents a very typical case for the spin contributions of the lower exciton states. To demonstrate this, in Figs. 9-12, we plot the spin contributions of the lower exciton states for QD2, QD11, QD5, and QD8 in the same way as in Fig. 5 for QD1. Note that for a better comparability the energy scale in all figures is the same. The elongation (QD2, Fig. 9) and the volume (QD11, Fig. 10) have nearly no impact on the overall behavior of the spin mixtures, even if for larger volumes the energy separations are strongly reduced. In contrast, the height shifts the relative position between $\mathrm{LH}$ and HH states. In very flat QDs (QD5, Fig. 11), the LH excitons are shifted to higher energies, which simultaneously reduces the spin mixtures of the lower levels. In a very high QD (QD8, Fig. 12), the LH excitons are at lower energies, and there is a larger number of states with strongly mixed spin-character already at low energies.

\section{APPENDIX C: TABLES}

To provide explicit values of our results presented in Figs. 3 and 6, we present two tables listing the state mixtures of the most relevant states (Tables I and II). The quantitatively given results are estimates within the limitations of our model. 
[1] P. Michler, Quantum Dots for Quantum Information Technologies (Springer International Publishing, Cham, 2017), Vol. 237.

[2] O. Gazzano and G. S. Solomon, Toward optical quantum information processing with quantum dots coupled to microstructures, J. Opt. Soc. Am. B 33, C160 (2016).

[3] P. Senellart, G. Solomon, and A. White, High-performance semiconductor quantum-dot single-photon sources, Nat. Nanotechnol. 12, 1026 (2017).

[4] D. Huber, M. Reindl, J. Aberl, A. Rastelli, and R. Trotta, Semiconductor quantum dots as an ideal source of polarizationentangled photon pairs on-demand: A review, J. Opt. 20, 073002 (2018).

[5] S. L. Portalupi, M. Jetter, and P. Michler, InAs quantum dots grown on metamorphic buffers as non-classical light sources at telecom C-band: A review, Semicond. Sci. Technol. 34, 053001 (2019).

[6] S. Rodt, S. Reitzenstein, and T. Heindel, Deterministically fabricated solid-state quantum-light sources, J. Phys.: Condens. Matter 32, 153003 (2020).

[7] E. Biolatti, R. C. Iotti, P. Zanardi, and F. Rossi, Quantum Information Processing with Semiconductor Macroatoms, Phys. Rev. Lett. 85, 5647 (2000).

[8] A. J. Ramsay, A review of the coherent optical control of the exciton and spin states of semiconductor quantum dots, Semicond. Sci. Technol. 25, 103001 (2010).

[9] R. J. Warburton, Single spins in self-assembled quantum dots, Nat. Mater. 12, 483 (2013).

[10] J. McFarlane, P. A. Dalgarno, B. D. Gerardot, R. H. Hadfield, R. J. Warburton, K. Karrai, A. Badolato, and P. M. Petroff, Gigahertz bandwidth electrical control over a dark exciton-based memory bit in a single quantum dot, Appl. Phys. Lett. 94, 093113 (2009).

[11] J. Johansen, B. Julsgaard, S. Stobbe, J. M. Hvam, and P. Lodahl, Probing long-lived dark excitons in self-assembled quantum dots, Phys. Rev. B 81, 081304(R) (2010).

[12] E. Poem, Y. Kodriano, C. Tradonsky, N. H. Lindner, B. D. Gerardot, P. M. Petroff, and D. Gershoni, Accessing the dark exciton with light, Nat. Phys. 6, 993 (2010).

[13] Y. H. Huo, V. Křápek, O. G. Schmidt, and A. Rastelli, Spontaneous brightening of dark excitons in GaAs/AlGaAs quantum dots near a cleaved facet, Phys. Rev. B 95, 165304 (2017).

[14] T. Heindel, A. Thoma, I. Schwartz, E. R. Schmidgall, L. Gantz, D. Cogan, M. Strauß, P. Schnauber, M. Gschrey, J. H. Schulze et al., Accessing the dark exciton spin in deterministic quantumdot microlenses, APL Photonics 2, 121303 (2017).

[15] S. Lüker and D. E. Reiter, A review on optical excitation of semiconductor quantum dots under the influence of phonons, Semicond. Sci. Technol. 34, 063002 (2019).

[16] D. E. Reiter, T. Kuhn, and V. M. Axt, All-Optical Spin Manipulation of a Single Manganese Atom in a Quantum Dot, Phys. Rev. Lett. 102, 177403 (2009).

[17] I. Schwartz, D. Cogan, E. R. Schmidgall, Y. Don, L. Gantz, O. Kenneth, N. H. Lindner, and D. Gershoni, Deterministic generation of a cluster state of entangled photons, Science 354, 434 (2016).

[18] S. Lüker, T. Kuhn, and D. E. Reiter, Direct optical state preparation of the dark exciton in a quantum dot, Phys. Rev. B 92, 201305(R) (2015).

[19] S. Germanis, P. Atkinson, R. Hostein, C. Gourdon, V. Voliotis, A. Lemaitre, M. Bernard, F. Margaillan, S. Majrab, and B.
Eble, Dark-bright exciton coupling in asymmetric quantum dots, Phys. Rev. B 98, 155303 (2018).

[20] M. Zieliński, Y. Don, and D. Gershoni, Atomistic theory of dark excitons in self-assembled quantum dots of reduced symmetry, Phys. Rev. B 91, 085403 (2015).

[21] I. Schwartz, E. R. Schmidgall, L. Gantz, D. Cogan, E. Bordo, Y. Don, M. Zielinski, and D. Gershoni, Deterministic Writing and Control of the Dark Exciton Spin Using Single Short Optical Pulses, Phys. Rev. X 5, 011009 (2015).

[22] T. Smoleński, T. Kazimierczuk, M. Goryca, T. Jakubczyk, Ł. Kłopotowski, Ł. Cywiński, P. Wojnar, A. Golnik, and P. Kossacki, In-plane radiative recombination channel of a dark exciton in self-assembled quantum dots, Phys. Rev. B 86 , 241305(R) (2012).

[23] J. Huneke, I. D’Amico, P. Machnikowski, T. Thomay, R. Bratschitsch, A. Leitenstorfer, and T. Kuhn, Role of Coulomb correlations for femtosecond pump-probe signals obtained from a single quantum dot, Phys. Rev. B 84, 115320 (2011).

[24] T. Suzuki, R. Singh, G. Moody, M. Aßmann, M. Bayer, A. Ludwig, A. D. Wieck, and S. T. Cundiff, Dephasing of InAs quantum dot p-shell excitons studied using two-dimensional coherent spectroscopy, Phys. Rev. B 98, 195304 (2018).

[25] C. Hinz, P. Gumbsheimer, C. Traum, M. Holtkemper, B. Bauer, J. Haase, S. Mahapatra, A. Frey, K. Brunner, D. E. Reiter, T. Kuhn, D. V. Seletskiy, and A. Leitenstorfer, Charge and spin control of ultrafast electron and hole dynamics in single CdSe/ZnSe quantum dots, Phys. Rev. B 97, 045302 (2018).

[26] M. Holtkemper, D. E. Reiter, and T. Kuhn, Influence of the quantum dot geometry on p-shell transitions in differently charged quantum dots, Phys. Rev. B 97, 075308 (2018).

[27] C. Qian, X. Xie, J. Yang, K. Peng, S. Wu, F. Song, S. Sun, J. Dang, Y. Yu, M. J. Steer et al., Enhanced Strong Interaction between Nanocavities and p-Shell Excitons Beyond the Dipole Approximation, Phys. Rev. Lett. 122, 087401 (2019).

[28] G. F. Quinteiro, D. E. Reiter, and T. Kuhn, Formulation of the twisted-light-matter interaction at the phase singularity: The twisted-light gauge, Phys. Rev. A 91, 033808 (2015).

[29] G. F. Quinteiro and T. Kuhn, Light-hole transitions in quantum dots: Realizing full control by highly focused optical-vortex beams, Phys. Rev. B 90, 115401 (2014).

[30] Y. H. Huo, B. J. Witek, S. Kumar, J. R. Cardenas, J. X. Zhang, N. Akopian, R. Singh, E. Zallo, R. Grifone, D. Kriegner et al., A light-hole exciton in a quantum dot, Nat. Phys. 10, 46 (2014).

[31] K. S. Youngworth and T. G. Brown, Focusing of high numerical aperture cylindrical-vector beams, Opt. Express 7, 77 (2000).

[32] L. Novotny, M. R. Beversluis, K. S. Youngworth, and T. G. Brown, Longitudinal Field Modes Probed by Single Molecules, Phys. Rev. Lett. 86, 5251 (2001).

[33] Q. Zhan, Trapping metallic rayleigh particles with radial polarization, Opt. Express 12, 3377 (2004).

[34] G. F. Quinteiro, F. Schmidt-Kaler, and C. T. Schmiegelow, Twisted-Light-Ion Interaction: The Role of Longitudinal Fields, Phys. Rev. Lett. 119, 253203 (2017).

[35] J. Durnin, J. J. Miceli, and J. H. Eberly, Diffraction-Free Beams, Phys. Rev. Lett. 58, 1499 (1987).

[36] M. Woerdemann, C. Alpmann, M. Esseling, and C. Denz, Advanced optical trapping by complex beam shaping, Laser Photon. Rev. 7, 839 (2013). 
[37] M. Ettorre, S. C. Pavone, M. Casaletti, and M. Albani, Experimental validation of Bessel beam generation using an inward Hankel aperture distribution, IEEE Trans. Antennas Propag. 63, 2539 (2015).

[38] G. Milione, A. Dudley, T. A. Nguyen, O. Chakraborty, E. Karimi, A. Forbes, and R. A. Alfano, Measuring the selfhealing of the spatially inhomogeneous states of polarization of vector Bessel beams, J. Opt. 17, 035617 (2015).

[39] S. Ninomiya and S. Adachi, Optical properties of cubic and hexagonal CdSe, J. Appl. Phys. 78, 4681 (1995).

[40] W. Chen and Q. Zhan, Realization of an evanescent Bessel beam via surface plasmon interference excited by a radially polarized beam, Opt. Lett. 34, 722 (2009).

[41] H. Huang, Q. Li, J. Fu, J. Wu, F. Lin, and X. Wu, Efficient subwavelength focusing of light with a long focal depth, Nanoscale 7, 16504 (2015)

[42] I. A. Merkulov, A. L. Efros, and M. Rosen, Electron spin relaxation by nuclei in semiconductor quantum dots, Phys. Rev. B 65, 205309 (2002).

[43] S. Trumm, M. Wesseli, H. J. Krenner, D. Schuh, M. Bichler, J. J. Finley, and M. Betz, Spin-preserving ultrafast carrier capture and relaxation in InGaAs quantum dots, Appl. Phys. Lett. 87, 153113 (2005).

[44] K. Gawarecki, M. Pochwała, A. Grodecka-Grad, and P. Machnikowski, Phonon-assisted relaxation and tunneling in self-assembled quantum dot molecules, Phys. Rev. B 81, 245312 (2010).
[45] T Grange, R Ferreira, and G Bastard, Polaron relaxation in self-assembled quantum dots: Breakdown of the semiclassical model, Phys. Rev. B 76, 241304(R) (2007).

[46] U. E. H. Laheld and G. T. Einevoll, Excitons in CdSe quantum dots, Phys. Rev. B 55, 5184 (1997).

[47] O. Madelung, Semiconductors: Data Handbook (Springer, Berlin, Heidelberg, 2012).

[48] M. R. Salvador, M. W. Graham, and G. D. Scholes, Excitonphonon coupling and disorder in the excited states of CdSe colloidal quantum dots, J. Chem. Phys. 125, 184709 (2006).

[49] R. M. Martin, Piezoelectricity, Phys. Rev. B 5, 1607 (1972).

[50] C. I. Contescu and K. Putyera, Dekker Encyclopedia of Nanoscience and Nanotechnology (CRC Press, Boca Raton, FL, 2009).

[51] M. Gorska and W. Nazarewicz, Application of the randomelement isodisplacement model to long-wavelength optical phonons in $\mathrm{CdSe}_{x} \mathrm{Te}_{1-x}$ mixed crystals, Phys. Status Solidi B 65, 193 (1974).

[52] H. Lange, M. Artemyev, U. Woggon, and C. Thomsen, Raman investigation of strain effects in CdSe nanorods, Phys. Status Solidi B 246, 2817 (2009).

[53] A. K. Arora, Grüneisen parameter of soft phonons and high pressure phase transitions in semiconductors, J. Phys. Chem. Solids 51, 373 (1990). 